\title{
Space Division Multiple Access Technology for Free Space Optical Communication
}

\author{
Jing Chen, Guangyin Xu, Jiandong Hu, Xiushan Wang, Ling Wang \\ College of Mechanical and Electrical Engineering, Henan Agricultural University, Zhengzhou, China \\ chenbaobaoguai@126.com
}

\begin{abstract}
Keywords: Free space optical communications; Optical switching devices; Fiber array; Space division multiplexing access.

Abstract. This project about how to implement space division multiplexing access technology in free space optical communication is presented. It works in fixed beams switching mode and consists of optical lens based on fiber array of focal plane and optical matrix switch. Based on angle, by setting up the gateway of optical switch, optical matrix switch actualizes the tracking receiving of user's beam and multi-beam directional transmission and continuous tracking. At the same time, Optical lens designed with big field of view by ZEMAX. The feasibility and performance of the coupling of lens was simulated study. It validates the qualitative feasibility of the technology.
\end{abstract}

\section{Introduction}

Free space optical (FSO) communication technology has made enormous and steady progress for several decades [1, 2], providing the new resource in our increasingly information-driven society and economy. Much of this progress has been in finding innovative ways to increase the data carrying capacity. Unlike in RF, by "channel”, we mean spatial channel rather than frequency channel. Multi-channel array design for FSO communication is very attractive since it offers high aggregate bandwidth due to spatial diversity.

The notion of increasing fiber capacity with Space Division Multiplexing Access (SDMA) is almost as old as fiber communications itself, with the fabrication of fibers containing multiple cores, the first and most obvious approach to SDMA, reported as far back as 1979 [3, 4]. Recently multicore fiber (MCF) and multimode fiber (MMF) approach has been given to building a complete networking platform [5, 6]. Yet SDMA technology was rarely published in FSO communication until now. Because the available wavelength resource in atmospheric window is deficient, and the smaller divergence angle brings difficulties on acquisition, tracking and pointing (ATP).

At present, FSO communications use a single transmitting antenna and a receiving antenna for single channel communication between two nodes [7, 8]. In this paper, we explore multi-channel FSO communication system using SDMA technology and research the feasibility of key technologies and implementation scheme.

\section{System description and channel model}

Multiple input optical lenses are the core component of SDMA in FSO communication. While entering the field of base station, user unilaterally acquires the lens of base station, and actualizes tracking, pointing meanwhile. Then, signal laser will be emitted in the direction of base station.

The functions of technology include space channels segmentation, multi-beam coordinative receiving, multi-beam forming and tracking.

Structurally, as shown in Fig. 1, it consists of optic lens with large field view, and fiber array on focal plane with displacement adjustment, optical matrix switch (OMS) and controller. Based on detection angle of Charge Coupled Device (CCD), by setting up the gateway of optical switch, the optical matrix switch actualizes the tracking receiving of multi-beam directional transmission and continuous tracking. Virtual channels segmented by fiber array on the focal plane of optical lens. The 
fiber array's structure is hexagonal. According to optical principle, one fiber corresponds to one fixed field of lens, or named one fixed subarea of cell. The uplink beam of user in that subarea receives by the fiber. The fiber's output port connects to the input port of optical matrix switch.

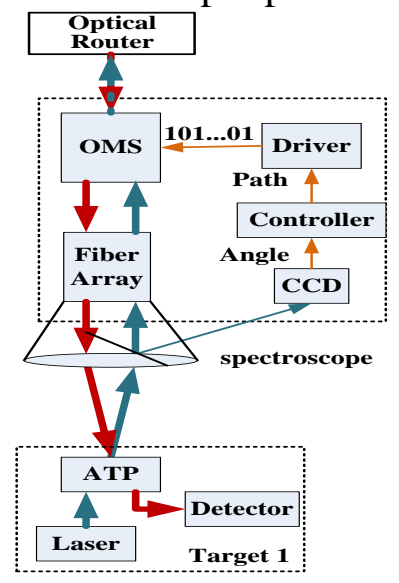

Fig.1.Stucture of multiple input optical lens

The working principle of single user tracking receiving is shown in Fig. 2. CCD sensor detects the angle of user's optical beam, calibrated by orbit parameters predefined. Imaging spot focus on is corresponding the fiber. Then input and output ( $\mathrm{I} / \mathrm{O})$ port was distributed for the user. When the controller of optical matrix switch opens the optical switches on the path, user's beam is switched to the $\mathrm{I} / \mathrm{O}$ port.

While user moving to another place, on the assumption that it's imaging spot was focus on fiber b. The controller of optical matrix switch just needs to open the optical switches on the path. The beam is switched to the same $\mathrm{I} / \mathrm{O}$ port.

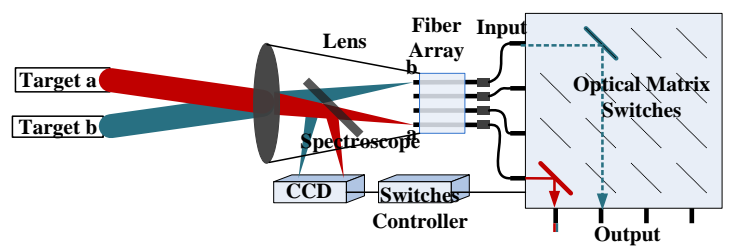

Fig.2. Sketch map of multiuser access

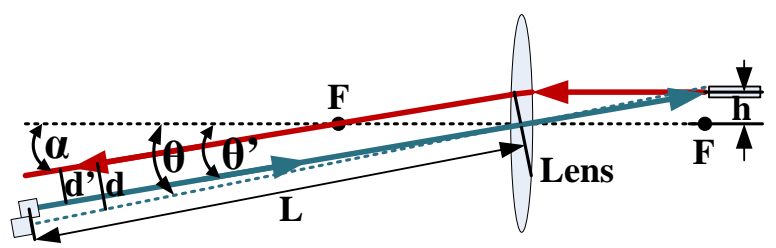

Fig.3 Working principle of multi-beam switching

Using motion estimation of digital image processing technology, it can estimate the user's next position, so as to pre-establish the next path. It is the prerequisite to realize continuous tracking receiving.

The working principle is that CCD sensor gets image of laser spots, and image segmentation and calibrating confirms the fibers that imaging spots are focus on respectively. As in signal user tracking receiving, distributes two fixed I/O ports for them. And the corresponding output ports of optical matrix switch are changed. Setting the path linked be changed, it switches user's beams to the I/O ports respectively.

While users are moving, the tracking method is same as single user tracking.

The working principle of multi-beam switching is shown in Fig. 3. As known to all, based on optics principle, user, lens center and image spot are in line. However the light paralleling with optical axes must emit through the focus.

However, based on geometrical optics, the angle $\alpha$ of one fiber is fixed. If known the angle $\theta$ or $\theta$ ' ( $\theta$ is the angle of beams focus on random position of fiber, and $\theta^{\prime}$ is the angle of beams focus on the center of fiber.), the distance L between user (corresponding to receiving fiber ' $a$ ') and base station, it can deduce the $\mathrm{d}$ which is the distance between uplink beam and downlink beam:

$$
d=L(\tan \theta-\tan \alpha)+f \times \tan \alpha
$$


If it designs that the radius of beam equals to or is bigger than "d", we can use the same fiber to transmit uplink beam and downlink beam. There is no processing on signal laser, so it can be used in all-optical communication.

\section{Simulation}

Taking lens designed with $2^{\circ}$ field of view as example by ZEMAX. We discuss the feasibility and performance of the coupling of lens and fiber array.

Thirty-seven fibers arrayed as a hexagon. All fiber-optic end faces are on a plane exactly by grinding. Fiber diameter with cladding is $125 \mu \mathrm{m}$; the core diameter is $105 \mu \mathrm{m}$; numerical-aperture is 0.22 . The diameter of fiber array end face is $875 \mu \mathrm{m}$, receiving field is $2^{\circ}$. So set the focus of lens at 25 $\mathrm{mm}$; entrance pupil at $10 \mathrm{~mm}$; wavelength at $1550 \mathrm{~nm}$.

The dimension of every lens is processed with former and off-focus. Spot diagram of lens is shown in Fig. 4. It reveals that the design of optical system nears diffraction limit and diameter of diffraction limit is $9.46 \mu \mathrm{m}$.

The FFT diffraction encircled energy of lens is shown in Fig. 5. It reveals that the energy encircled by fiber core approaches to $99 \%$. The lens is provided with good performance of beam focusing.

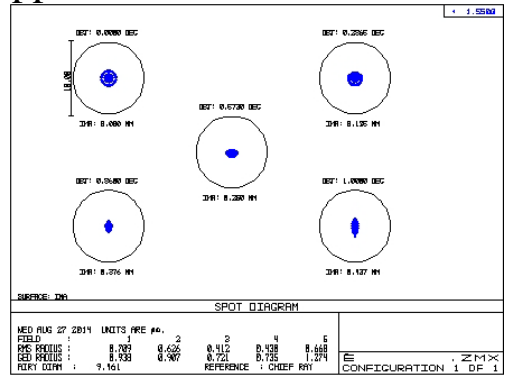

Fig.4 Spot diagram of lens analyzed

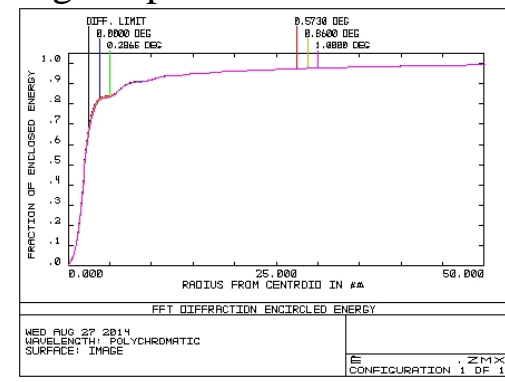

Fig.5 FFT diffraction en circled energy of lens analyzed

Energy distribution of different field ((a) $0.57^{\circ}$, (b) $0.86^{\circ}$ ) is shown in Fig. 6. It reveals that near the field center, the spot is near symmetrical; in border field, energy distribution is a symmetrica land off-centered which is due to optical aberration. However, the spot diameters are all less than $2.5 \mu m$; the image numerical-aperture also is smaller than 0.22. In the point of design, optical aberration has little effect on the coupling with fiber.

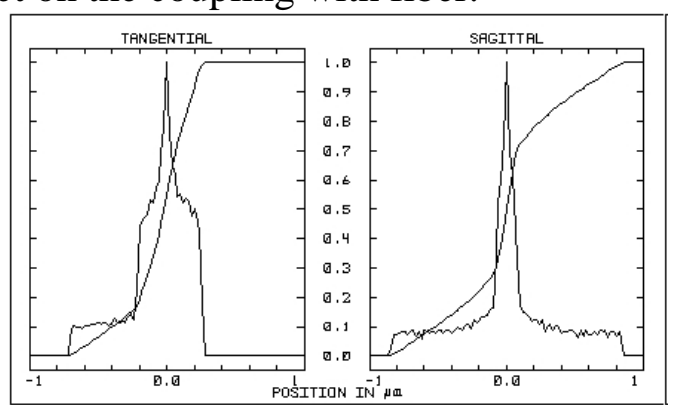

(a)

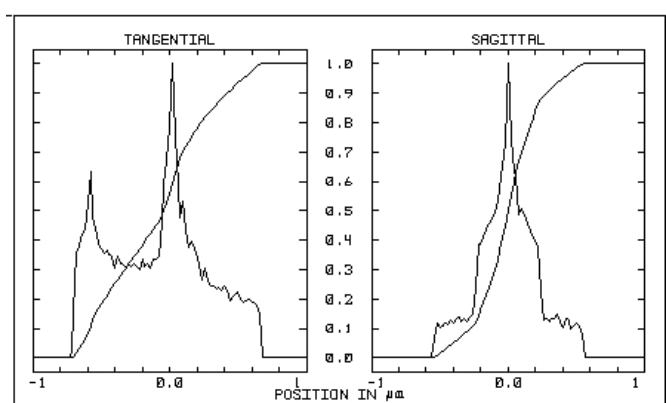

(b)

Fig.6 Energy distribution of border field analyzed

Angular resolution is codetermined by optical lens and focal plane device. Optical lens resolution equation is following:

$$
\alpha=1.22 \lambda / D
$$

The focal plane device is fiber array, its academic angular resolution determined by fiber diameter $\mathrm{R}^{\prime}$ and focus $\mathrm{f}$ :

$$
\beta=R^{\prime} / f
$$


It reveals that angular resolution of the system is restricted by fiber diameter.

Setting the field on $0^{\circ}, 0.29^{\circ}, 0.57^{\circ}, 0.86^{\circ}$, and $1^{\circ}$, we get the image footprint diagram of lens as shown in Fig. 7. It reveals that except $1^{\circ}$, all beams in other field are focus on the fibers center respectively. The beam in $1^{\circ}$ field focuses on the border of two fibers. It will confuse the beam in $0.86^{\circ}$ field with the beam in $1^{\circ}$ field.

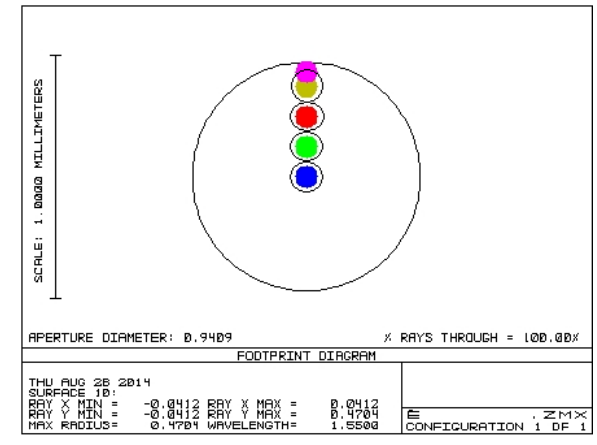

Fig.7 Footprint diagram of lens analyzed

According to the above analysis, the design of taking fiber array as focal plane device is feasible. And it can meet the need of virtual channel segmentation.

\section{Conclusions}

We demonstrated that SDMA technology for free-space optical (FSO) communications is feasible. It will give excellent bandwidth performance in future. In this paper, we considered designs for the optical fiber arrays based on focal plane and optical matrix switch for analysis. Optical simulation preliminarily proved that the technology is feasible in optical system. An interesting future problem is to find an optimal design for the array that achieves highest capacity for a given range, transmitter divergence, and the number of transmitters.

\section{References}

[1] I. Djordjevic, "Deep-space and near-Earth optical communications by coded orbital angular momentum (OAM) modulation,” Opt. Express 19, 14277-14289 (2011).

[2] A. Sevincer, M. Bilgi, and M. Yuksel, "Automatic realignment with electronic steering of free-space-optical transceivers in MANETs: A proof-of-concept prototype,” Ad Hoc Networks, vol. 11, no. 1, p.585-595, (2013).

[3] Inao, S. et al. Multicore optical fiber. Proc.Optical Fiber Communications Conference, WB1, 46-8 (1979).

[4] D. J. Richardson, J. M. Fini, L E. Nelson .Space Division Multiplexing in Optical Fibres. Nature Photonics 7, p.354-362 (2013)

[5] Sillard, P. New fibers for ultra-high capacity transport. Opical Fiber Technology 17, 495-502, (2011).

[6] Wheeler N. V. et al. Wide-bandwidth, low-loss19-cell hollow core photonic band gap fiber and its potential for low latency transmission. Proc. OFC/NFOEC 2012, paper PDP5A.2 (2012).

[7] Jayasri Akella, Murat Yuksel, Shiv Kalyanaraman. Multi-channel Communication in Free-Space Optical Networks for the Last-mile. IEEE Workshop on Local and Metropolitan Area Networks,43-49 (2007).

[8] B. Wu, P.-H. Ho, K. L. Yeung, J. Tapolcai, and H. T. Mouftah, "Optical layer monitoring schemes for fast link failure localization in all-optical networks," IEEE Commun. Surveys Tutorials, vol. 13, no. 1, p. 114-125, (2011). 